Bio - grafía. Escritos sobre la Biología y su Enseñanza. ISSN 2027-1034

Edición Extraordinaria. p.p. 1052 - 1059

Memorias del IX Encuentro Nacional de Experiencias en Enseñanza de la Biología y la Educación Ambiental. IV Congreso Nacional de Investigación en Enseñanza de la Biología.

\title{
INTERDISCIPLINARIEDAD CURRICULAR. UNA PROPUESTA TRABAJANDO CON INSECTOS EN GRADO QUINTO DE PRIMARIA
}

\author{
Gamboa, Camila \& Mondol, Sharon ${ }^{1}$ \\ Valbuena Ussa, Edgar ${ }^{2}$
}

\section{RESUMEN}

En esta ponencia se presentan los resultados parciales del trabajo de grado "Los insectos como estrategia de enseñanza interdisciplinar una propuesta pedagógica con maestros del grado quinto de un colegio público de Bogotá". El estudio se ha realizado en la perspectiva de la investigación acción pedagógica, implicando a cuatro profesores de distintas áreas curriculares de la institución. Los datos de investigación se obtuvieron a partir de observaciones no participantes de sesiones de clases, programas curriculares, documentos institucionales, entrevistas no estructuradas, y sesiones con el equipo de profesores.

Los principales elementos potenciadores de interdisciplinariedad identificados por el equipo son, las necesidades comunes de formar en valores (convivencia, respeto, tolerancia y responsabilidad) y mejorar la comunicación de los estudiantes; el abordaje de contenidos formativos específicos de cuatro áreas curriculares (lengua castellana, matemáticas, tecnología y ciencias naturales) mediante el trabajo con insectos (producción de textos, expresión oral, análisis de problemas matemáticos, utilización de las TIC, y biofilia).

PALABRAS CLAVE: Interdisciplinariedad curricular, insectos, investigación acción pedagógica, reflexión docente.

\section{ABSTRACT:}

This paper presents the partial results of the work of degree "Insects as an interdisciplinary teaching strategy a pedagogical proposal with teachers of the fifth grade of a public school in Bogotá." The study was carried out in the perspective of research pedagogical action, involving four teachers from different curricular areas of the educational institution. The research data were obtained from non-participant observations of class sessions, curricular programs, institutional documents, unstructured interviews, and sessions with the faculty team.

\footnotetext{
${ }^{1}$ Estudiantes Universidad Pedagógica Nacional. Departamento de Biología. e-mail: camila ; sharonmondol@hotmail.com anacamilagamboabarragan@gmail.com

2 Profesor Universidad Pedagógica Nacional. Coordinador Grupo de Investigación Conocimiento Profesional del Profesor de Ciencias.e-mail: valbuena@pedagogica.edu.co , edgarorlay@hotmail.com
} 
Bio - grafía. Escritos sobre la Biología y su Enseñanza. ISSN 2027-1034

Edición Extraordinaria. p.p. 1052 - 1059

Memorias del IX Encuentro Nacional de Experiencias en Enseñanza de la Biología y la Educación Ambiental. IV Congreso Nacional de Investigación en Enseñanza de la Biología.

The main enhancing elements of interdisciplinarity identified by the team are the common needs of forming in values (coexistence, respect, tolerance and responsibility) and improve student communication; (text production, oral expression, analysis of mathematical problems, use of ICTs, and biophysics). In this paper we present the results of the study of four different curricular areas: Spanish, mathematics, technology and natural sciences.

KEY WORDS: Curricular interdisciplinarity, insects, research pedagogical action, teacher reflection.

\section{INTRODUCCIÓN}

La presente investigación surge del trabajo de grado titulado "Los insectos como estrategia de enseñanza interdisciplinar una propuesta pedagógica con maestros del grado quinto de la IED Francisco de Miranda" se hila a través del proceso de formación del programa de pregrado de Licenciatura en Biología de la Universidad Pedagógica Nacional, basándose en las realidades de la escuela, que fueron vivenciadas en el transcurso de la práctica pedagógica durante el año 2016 de las maestras en formación; al igual que, en los distintos acercamientos a instituciones educativas durante el proceso formativo a lo largo de la licenciatura. Debido a lo anterior, emergen diferentes percepciones en cuanto a la enseñanza-aprendizaje, las cuales surgen de experiencias dentro del proceso de formación, donde, se evidencia una preocupación frente a la incorporación de la enseñanza interdisciplinar, lo cual representa un nuevo reto en la educación, trayendo consigo la inclusión de nuevas temáticas en la malla curricular de cada grado.

De cierto modo la interdisciplinariedad amplía el espectro o alcance de los grupos de trabajo, permitiéndose que se complemente de mejor manera el desarrollo de ciertos ejes temáticos, de tal manera que es posible afirmar que el estudiante formado con interdisciplinariedad podrá abarcar de mejor forma cada situación que se le presentase, tanto a nivel educativo como profesional y personal.

"La interdisciplinariedad, al contrario, incorpora los resultados de las diversas disciplinas, tomándolas de los diferentes esquemas conceptuales de análisis, sometiéndose a comparación y enjuiciamiento y, finalmente, integrandolas" (Tamayo, 2011, p. 5)

Este proceso investigativo se está desarrollando con la participación de los profesores de grado quinto del colegio ubicado en la localidad octava (Kennedy), donde se desarrolla junto a ellos una propuesta pedagógica que vincule las áreas de matemáticas, lengua castellana, tecnología y ciencias naturales, alrededor de una misma temática la cual fue la clase insecta, esto con el fin de implementar estrategias de aprendizaje que fueran innovadoras en el salón y así mismo dinamizar las clases, teniendo como método de trabajo la investigación acción pedagógica. Se decide trabajar la propuesta desde una mirada interdisciplinar con un enfoque de aprendizaje significativo, en vista que se asocian las áreas de manera integral de tal manera que el estudiante vincule los conocimientos adquiridos con su entorno cotidiano y no aislé el conocimiento en su diario vivir. Desde la línea de investigación de Conocimiento 
Bio - grafía. Escritos sobre la Biología y su Enseñanza. ISSN 2027-1034

Edición Extraordinaria. p.p. 1052 - 1059

Memorias del IX Encuentro Nacional de Experiencias en Enseñanza de la Biología y la Educación Ambiental. IV Congreso Nacional de Investigación en Enseñanza de la Biología.

Profesional Del Profesor De Ciencias (CPPC), se incentiva a replantear prácticas discursivas que den un nuevo aire al sistema educativo desde su formación y experiencia, creando propuestas que tiendan a la interdisciplinariedad entre áreas, para que el maestro no solo se involucre desde su área de conocimiento, sino que, pueda disponerse a otros panoramas, creando nuevos saberes que se irán generando en el transcurso del proceso y la experiencia educativa.

\section{METODOLOGÍA}

Este estudio se aborda desde el enfoque de investigación acción pedagógica, se escogió esta metodología de trabajo teniendo en cuenta el nivel de flexibilidad que se debe manejar a la hora de trabajar con un grupo de maestros, puesto que los tiempos y las ocupaciones de los mencionados son reducidos y hay que comprender que es una ardua jornada de trabajo la que realizan. Para trabajar con los maestros, se asignó un espacio, en el horario de 11:30 am a 12:30 pm después de la jornada escolar en la sala de sistemas, donde, los docentes usan este espacio para la reflexión y el trabajo en conjunto, haciendo las planeaciones y modificaciones que se generan a partir del transcurso de las clases que retroalimentaron la propuesta.

Se retoma el término investigación-acción el cual se le atribuye al psicólogo Kurt Lewin, quien lo utilizó para designar el método de varios de sus trabajos de intervención comunitaria durante la segunda guerra mundial y pocos años después. El interés primario de Lewin no era describir una situación social conflictiva; tampoco interpretarla o extraer conclusiones teóricas, a fin de proponer leyes de conducta y evolución social. Su objetivo era mejorar la situación estudiada y hacerlo precisamente mientras investigaba. Esto supone la necesidad de actuar, intervenir activamente en la comunidad, probar soluciones y observar los resultados (Parra, 2002). Como se resalta en el documento.

"No queremos acción sin investigación, ni investigación sin acción", puesto que se pueden determinar circunstancias a través de una acción concreta y aprender algo acerca de aquello sobre lo que se ha ejercido la acción. Se trata en gran modo de un aprendizaje práctico que puede basarse desde la experiencia para intervenir en un grupo de estudiantes. (Kurt Lewin, citado en Parra, 2002, p115).

Por lo tanto la investigación surge alrededor de la integración en cuanto a la interdisciplinariedad la cual se ha convertido en el tema de debate en la educación actual, donde el maestro debe trascender en la educación de sus estudiantes, convirtiendo este saber a futuro en aprendizaje significativo. Es por ello que desde su experiencia el maestro está expuesto a distintos desafíos donde utilizará todo lo que ha desarrollado durante su faceta en formación, concibiéndose como un investigador activo en el salón de clases el cual problematiza falencias que se perciben en los estudiantes, para buscar posibles soluciones en pro de la educación, donde el maestro investigador es actor en el proceso de su proyecto, formulando, desarrollando y evaluando su trabajo durante la práctica, reconociendo en gran medida su rol e importancia en la sociedad. Este proceso está acompañado de las siguientes fases de investigación: 
Bio - grafía. Escritos sobre la Biología y su Enseñanza. ISSN 2027-1034

Edición Extraordinaria. p.p. 1052 - 1059

Memorias del IX Encuentro Nacional de Experiencias en Enseñanza de la Biología y la Educación Ambiental. IV Congreso Nacional de Investigación en Enseñanza de la Biología.
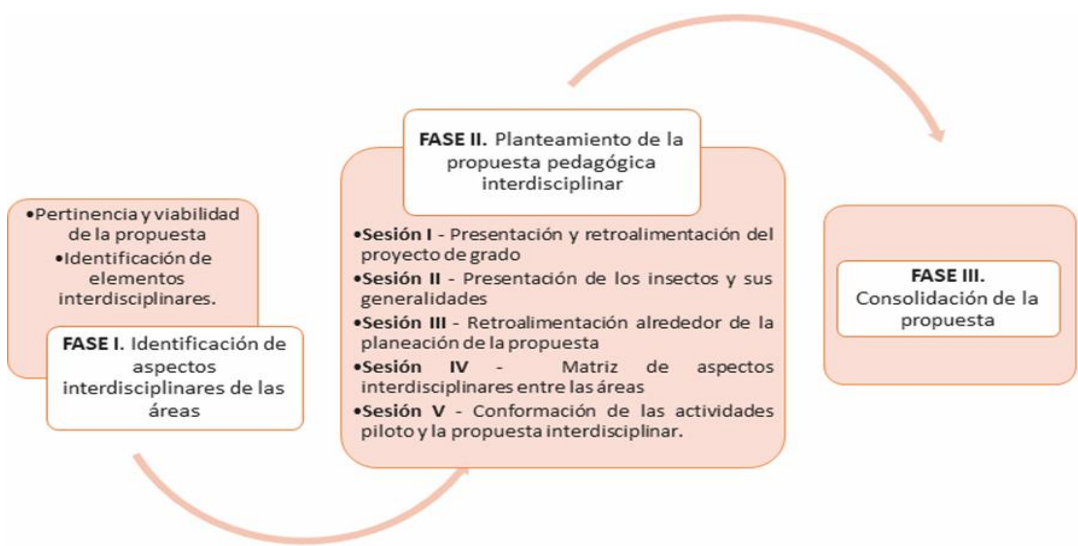

Esquema propio. Fases de la investigación.

Como se mencionó anteriormente, la investigación consta de tres fases, hasta el momento se han implementado la fase uno y la fase dos. La primera fase denominada identificación de los aspectos interdisciplinares de las áreas; en esta fase se realizaron una serie de filmaciones de audio y video de las sesiones de clase del área de Lengua Castellana, Matemáticas, tecnología y ciencias naturales; de las filmaciones y la observación se extrajeron elementos formativos y los contenidos de enseñanza que se evidencian durante las clases, al tener estos dos elementos, se comienzan a extraer los contenidos interdisciplinares que se lograron encontrar en cada área. En esta fase también se han incluido los documentos correspondientes al proyecto de aula y el PEI, de estos dos documentos también se extrajeron los contenidos formativos, de enseñanza e interdisciplinariedad. Se tomaron en cuenta estos dos documentos en vista que la propuesta emerge no sólo desde las mallas curriculares de las áreas, sino también los diferentes estamentos que se manejan dentro de la institución.

La segunda fase denominada Planteamiento de la propuesta interdisciplinar, aún está en ejecución, esta se encuentra dividida en varias sesiones, las cuales se trabajan de forma conjunta con el grupo focal, conformado por cuatro maestros uno por cada área de lengua castellana, matemáticas, tecnología y ciencias naturales, estas sesiones están destinadas para ser un espacio para compartir, discutir y llegar a acuerdos frente a los diferentes avances y sugerencias que se tienen para la construcción de la propuesta interdisciplinar, además de organizar actividades que permitan comprender algunos aspectos que sean importantes, por ejemplo, en una de las sesiones se habló sobre la clase insecta, con la finalidad de que los profesores identificara que organismos pertenecen a esta clase y que los caracteriza.

\section{RESULTADOS PARCIALES}

Dentro de los resultados que se han obteniendo en el transcurso de la investigación, se destacan los siguientes. El primer resultado, se obtiene en la primera fase de la investigación, la cual tuvo como objetivo indagar aquellos elementos de corte interdisciplinar que pudieran darle cuerpo a la propuesta; de esta indagación emergen tres categorías 
Bio - grafía. Escritos sobre la Biología y su Enseñanza. ISSN 2027-1034

Edición Extraordinaria. p.p. 1052 - 1059

Memorias del IX Encuentro Nacional de Experiencias en Enseñanza de la Biología y la Educación Ambiental. IV Congreso Nacional de Investigación en Enseñanza de la Biología.

- Contenidos formativos: Hacen referencia a la formación del estudiante como sujeto social el cual está permeado por diferentes dinámicas tanto dentro como fuera del colegio.

- Contenidos de enseñanza: hacen referencia a aquellas temáticas que se trabajan dentro de cada área y que podrĺan convertirse en temáticas interdisciplinares.

- Contenidos interdisciplinares: Estos son la selección de los dos anteriores, en esta categoría se agrupan tanto los contenidos formativos como de enseñanza que permiten integrar las cuatro áreas que se están trabajando, además se incluyen los documentos institucionales, los planes de área y la retroalimentación del equipo de trabajo.

La tabla 1 muestra las tres categorías, y los elementos que se encontraron en la primera fase de la investigación.

Tabla 1: Categorías emergentes en la fase 1

\begin{tabular}{|c|c|c|c|c|}
\hline $\begin{array}{l}\text { Categoría/ } \\
\text { área }\end{array}$ & $\begin{array}{c}\text { Lengua } \\
\text { Castellana }\end{array}$ & Matemáticas & Tecnología & $\begin{array}{l}\text { Ciencias } \\
\text { Naturales }\end{array}$ \\
\hline $\begin{array}{l}\text { Contenidos } \\
\text { Formativos }\end{array}$ & $\begin{array}{c}\text { Desarrollo de } \\
\text { procesos } \\
\text { cognitivos, } \\
\text { creativos y } \\
\text { socioafectivos. } \\
\text { Formar a los } \\
\text { estudiantes en una } \\
\text { educación general } \\
\text { fundamentada en } \\
\text { valores, } \\
\text { comunicación y } \\
\text { participación } \\
\text { activa. }\end{array}$ & $\begin{array}{c}\text { Capacidad de } \\
\text { abstraer las } \\
\text { matemáticas de su } \\
\text { diario vivir, para } \\
\text { lograr describir, el } \\
\text { aprendizaje está } \\
\text { interrelacionado } \\
\text { con las creencias, } \\
\text { con las } \\
\text { apreciaciones. }\end{array}$ & $\begin{array}{l}\text { Entusiasmo y } \\
\text { trabajo en equipo. } \\
\text { Formación en } \\
\text { robótica y uso de } \\
\text { las tics. }\end{array}$ & $\begin{array}{c}\text { La expresión y } \\
\text { la } \\
\text { comunicación } \\
\text { usadas como } \\
\text { una } \\
\text { herramienta } \\
\text { para comunicar } \\
\text { resultados de } \\
\text { los } \\
\text { investigaciones } \\
\text { generadas en } \\
\text { el proceso de } \\
\text { formación. }\end{array}$ \\
\hline $\begin{array}{l}\text { Contenidos } \\
\text { de } \\
\text { Enseñanza }\end{array}$ & $\begin{array}{l}\text { Buen uso de la } \\
\text { palabra en público } \\
\text { (oratoria), } \\
\text { capacidad de } \\
\text { sintetizar } \\
\text { información } \\
\text { mediante } \\
\text { resúmenes, } \\
\text { relación y solución } \\
\text { de problemas }\end{array}$ & $\begin{array}{l}\text { Interpretación de } \\
\text { fenómenos } \\
\text { naturales desde } \\
\text { modelos } \\
\text { matemáticos. } \\
\text { solidez de las } \\
\text { hipótesis, su } \\
\text { interpretación y las } \\
\text { conclusiones que } \\
\text { se elaboran }\end{array}$ & $\begin{array}{c}\text { Relacionar el } \\
\text { conocimiento } \\
\text { científico con el } \\
\text { conocimiento } \\
\text { tecnológico,identifi } \\
\text { cando el rol } \\
\text { ecológico para } \\
\text { integrarlo en esta } \\
\text { medida a robots } \\
\text { hechos a partir de } \\
\text { la morfología de }\end{array}$ & $\begin{array}{c}\text { Cuestionamient } \\
\text { o, formulación } \\
\text { de hipótesis y } \\
\text { explicitación de } \\
\text { teorías, donde } \\
\text { se utiliza el } \\
\text { respeto por los } \\
\text { otros para la } \\
\text { colaboración de } \\
\text { las soluciones } \\
\text { a dichos }\end{array}$ \\
\hline
\end{tabular}


Bio - grafía. Escritos sobre la Biología y su Enseñanza. ISSN 2027-1034

Edición Extraordinaria. p.p. 1052 - 1059

Memorias del IX Encuentro Nacional de Experiencias en Enseñanza de la Biología y la Educación Ambiental. IV Congreso Nacional de Investigación en Enseñanza de la Biología.

\begin{tabular}{|c|l|c|c|}
\hline & desde otras áreas. & los insectos. & problemas. \\
\hline \multirow{1}{*}{$\begin{array}{c}\text { Contenidos } \\
\text { Interdiscipli } \\
\text { nares }\end{array}$} & $\begin{array}{l}\text {-Resumen, cuento, oralidad, solución de problemas cotidianos, construcción y } \\
\text { producción de textos, respeto por los miembros de su entorno, colaboración, } \\
\text { tolerancia, contenidos más prácticos y que salgan de lo teórico, realización de } \\
\text { lectura periódica en distintos espacios de otras áreas donde se estima el } \\
\text { fortalecimiento de lecto-escritura y oralidad. Promueven la comunicación que } \\
\text { es un objetivo clave en todos los componentes institucionales y se relaciona de } \\
\text { manera integral en cada espacio académico. } \\
\text { ve el estudiante en un medio y cuales son sus interrelaciones. -Problemas } \\
\text { cotidianos para hacer la relación teoría-práctica, análisis de imágenes, textos. } \\
\text { Trabajo del pensamiento sistémico, cuestionamiento continuo. Se incentiva los } \\
\text { valores en cada una de las clases concibiendo al estudiante como un ser que } \\
\text { se comunica de forma afectiva y efectiva con otros miembros de la institución. }\end{array}$ \\
\hline
\end{tabular}

Estas tres categorías fueron el punto de partida para la segunda fase, ya que, al tener estos aspectos, se logra delimitar las temáticas. Como se mencionó en el apartado metodológico, la segunda fase consta de seis sesiones. Estas sesiones permitieron delimitar los siguientes elementos: En la tabla 2, se muestran los elementos interdisciplinares que tienen que ver con los contenidos de enseñanza, que han sido consolidados por el grupo de trabajo y sobre los cuales se construirá la propuesta

Tabla 2: Contenidos interdisciplinares propuestos por los docentes de las áreas

\begin{tabular}{|c|c|c|c|}
\hline $\begin{array}{c}\text { LENGUA } \\
\text { CASTELLANA }\end{array}$ & MATEMATICAS & TECNOLOGIA & $\begin{array}{c}\text { CIENCIAS } \\
\text { NATURALES }\end{array}$ \\
\hline $\begin{array}{c}\text { Descripción de } \\
\text { sucesos }\end{array}$ & $\begin{array}{c}\text { Noción de } \\
\text { cantidad } \\
\text { Potenciación }\end{array}$ & $\begin{array}{c}\text { Manejo de power } \\
\text { point }\end{array}$ & $\begin{array}{c}\text { Seres vivos } \\
\text { pluricelulares }\end{array}$ \\
\hline $\begin{array}{c}\text { Desarrollo de } \\
\text { textos }\end{array}$ & $\begin{array}{c}\text { Medidas de } \\
\text { longitud, área y } \\
\text { volumen }\end{array}$ & $\begin{array}{c}\text { Construcción de } \\
\text { vídeos, manejo } \\
\text { de la oralidad }\end{array}$ & $\begin{array}{c}\text { Cuidado del } \\
\text { ambiente }\end{array}$ \\
\hline $\begin{array}{c}\text { Comprensión de } \\
\text { lectura }\end{array}$ & $\begin{array}{c}\text { Geometría y } \\
\text { Estadísticas }\end{array}$ & $\begin{array}{c}\text { Buen uso de la } \\
\text { tic's }\end{array}$ & $\begin{array}{c}\text { Ecología, relación } \\
\text { planta-animal. }\end{array}$ \\
\hline
\end{tabular}


Bio - grafía. Escritos sobre la Biología y su Enseñanza. ISSN 2027-1034

Edición Extraordinaria. p.p. 1052 - 1059

Memorias del IX Encuentro Nacional de Experiencias en Enseñanza de la Biología y la Educación Ambiental. IV Congreso Nacional de Investigación en Enseñanza de la Biología.

\begin{tabular}{|c|c|c|c|}
$\begin{array}{c}\text { Buen uso de la } \\
\text { palabra en } \\
\text { público - oralidad }\end{array}$ & $\begin{array}{c}\text { Resolución de } \\
\text { problemas } \\
\text { cotidianos }\end{array}$ & $\begin{array}{c}\text { Creación de } \\
\text { insectos en } \\
\text { cuanto a robótica }\end{array}$ & $\begin{array}{c}\text { Relación con otros } \\
\text { organismos en el } \\
\text { diario vivir }\end{array}$ \\
\hline
\end{tabular}

Dentro de esta misma forma, se escogieron los elementos interdisciplinares formativos: Convivencia, respeto, tolerancia y responsabilidad, ya que estos son los que hay que potenciar en los alumnos. Para trabajar tanto los contenidos de enseñanza y los formativos interdisciplinares, se eligieron con el grupo de maestros cinco insectos: El piojo, la abeja, el cucarrón de mayo y los mosquitos, estos se eligieron teniendo en cuenta la posibilidad de verlos en el colegio, también de acuerdo a la relación con los elementos interdisciplinares mencionados anteriormente. Al iniciar la fase II, la cual se desarrolla con el apoyo de los docentes, se continúa el avance en la elaboración de la propuesta interdisciplinar, aquí la participación de los docentes es de vital importancia pues presentaron sus aportes a la estructura de la propuesta, las dudas que se suscitan en las sesiones hacen que la propuesta interdisciplinar se encamine a la enseñanza del estudiante en el área específica y también a la enseñanza de cada temática valiéndose del apoyo de una o varias áreas consideradas como las mejores para iniciar el trabajo.

Hubo un trabajo en conjunto con los maestros y otro individual de las maestras en formación, ya que las sesiones debían llevar un hilo conductor tanto con los aportes que los maestros habían hecho, como lo que surgía en el trabajo y consolidación de la propuesta interdisciplinar. Las sesiones fueron guiadas a la retroalimentación de los temas planteados en los planes de área, proyectos, clases y por los mismos maestros, con esta informacion se comenzó a tejer la propuesta donde se definieron tiempos de realización, posibles pruebas piloto con los estudiantes, temas tratados en las clases de cada uno y como podría ser la integración de los temas para que no solo quedara en la institución si no que trascendiera a sus casas buscando en gran medida la educación integral en sus estudiantes al reconocer la importancia del aprendizaje.

\section{CONCLUSIONES PARCIALES}

-La investigación acción pedagógica, no solo permite que el grupo focal intervenga de forma directa, sino que hace del trabajo una construcción conjunta, posibilitando que la construcción de la propuesta se enriquezca desde diferentes puntos de vista.La reflexión que suscita la práctica del maestro, permite que este encuentro varios elementos que no solo aporten a la construcción de la propuesta, sino, a la experiencia que poseen sus otros pares, pues, no todos vemos la vida del mismo modo y se nos pueden escapar detalles que otros si perciben y que son relevantes en nuestro vivir.

-Los maestros mostraron gran preocupación hacia la falta de sentido de pertenencia, el poco interés de aprender de sus estudiantes, y la segregación familiar que era considerada un punto clave en las dificultades anteriormente nombradas; con ello también se dieron a conocer frustraciones en cuanto que los maestros no sabían de qué manera llegar a sus estudiantes para que interpretaran sus acciones en la comunidad y cómo podrían cambiar dichas faltas. Las sesiones sirvieron de esparcimiento y diálogo de saberes como profesores y como personas principalmente, 
Bio - grafía. Escritos sobre la Biología y su Enseñanza. ISSN 2027-1034

Edición Extraordinaria. p.p. 1052 - 1059

Memorias del IX Encuentro Nacional de Experiencias en Enseñanza de la Biología y la Educación Ambiental. IV Congreso Nacional de Investigación en Enseñanza de la Biología.

donde se incentivo en todo momento que los estudiantes retoman interés por el conocimiento y como ciudadano, el cual se ve expuesto a interactuar constantemente con otros seres, y empezar desde algo pequeño como los insectos creará conciencia hacia diversas formas de la vida y lo vivo al mismo tiempo que se vinculan acciones de sus áreas al diario vivir.

\section{BIBLIOGRAFÍA}

Parra, C (2002). Investigación - Acción y desarrollo profesional. Educación y educadores, №5. Universidad de la Sabana. Colombia - Bogotá. Documento pdf. Recuperado de: http://www.redalyc.org/pdf/834/83400510.pdf

Restrepo, B (2004). La investigación-acción educativa y la construcción de saber pedagógico. Educación y Educadores, núm. 7. Universidad de La Sabana. Colombia. Documento pdf. Recuperado de: http://www.redalyc.org/pdf/834/83400706.pdf

Rivera (2006). La importancia de la interdisciplinariedad, reflexión desde un comunicado de la AMC. Recuperado el 9 de octubre de 2016, de http://www.edgarmorin.org/blog/46-ciencias-de-la-complejidad/624-la-

importancia-de-la-interdisciplinariedad-reflexion-desde-un-comunicado-de-laamc.htm

Tamayo, M (2011). La interdisciplinariedad. Universidad ICESI. Serie Cartillas Para el Docente. Publicaciones del CREA. 